\title{
Exosomal circRNAs as novel potential biomarkers for colorectal adenoma
}

\author{
Shaobing CHENG, Sen LU, Fanlong LIU* \\ Department of Colorectal Surgery, The First Affiliated Hospital, Zhejiang University School of Medicine, Hangzhou, Zhejiang, China \\ ${ }^{*}$ Correspondence: fanlong_liu@zju.edu.cn
}

Received July 18, 2021 / Accepted August 25, 2021

\begin{abstract}
Growing evidences have revealed that exosomal miRNAs, lncRNAs, and circRNAs play a pleiotropic role in tumor biology. Cell-cell communication mediated by exosomes has been considered to be a key factor in the malignant progression of colorectal cancer. However, the importance of exosome-derived circRNAs in the biological function and clinical significance of colorectal adenoma remains elusive. In this study, we aimed to identify altered circRNA expression profiles in exosomes isolated from plasma of patients with colorectal adenoma using high-throughput sequencing. Exosomes were confirmed by western blotting, transmission electron microscopy, and NanoSight assay. The sequencing data indicated that there are 413 differentially expressed circRNAs including 112 upregulated and 301 downregulated circRNAs in colorectal adenoma patients compared with controls. GO analysis and the circRNA-miRNA-mRNA network were performed to predict the potential function of circRNAs, and demonstrate the putative mechanisms in colorectal adenoma. Collectively, our findings revealed that plasma exosomal circRNAs may be a potential noninvasive biomarker for the detection of colorectal adenoma, and provided new insights into colorectal adenoma-carcinoma sequence.
\end{abstract}

Key words: circRNAs, exosome, colorectal adenoma, plasma, high-throughput sequencing

Colorectal cancer (CRC) is the most prevalent cancer and the second leading cause of cancer-related death [1]. The majority of colorectal adenomas are thought to be the precursor lesions to CRC. Over time, colorectal adenomas increase in size, develop increasingly dysplastic characteristics, and can eventually acquire invasive potential. The process of sequential alterations takes about 8-15 years [2]. The stepwise progression varies depending on the different molecular mechanisms. The screening programs is gradually popularization, especially endoscopic examination, many high-risk adenomas can be found at the time. Colonoscopy is the main screening method of the colon and rectum, which provides visualization of the entire large intestine, and can be used for localization, biopsy, and resection of a potential precancerous lesion [3]. The disadvantage of this method is an invasive operation, which is uncomfortable, and bowel preparation required drinking a lot of laxatives is also unpleasant. If it is an experienced expert to do the inspection, the cost will be higher [4]. There are other examinations, methods, including stool testing, blood testing, radiological examination, and so on $[5,6]$. Therefore, more affordable, minimally invasive, and non-invasive approaches are much needed to improve the overall sensitivity and patient compliance for colorectal adenoma screening.
Circular RNAs (circRNAs) are newly discovered non-coding RNAs, which have a closed circular structure without 5'caps and 3'poly tails [7-10]. The unique structure of circRNAs can resist the degradation of RNase R, making it more stable than linear parent genes [11-13]. CircRNAs have features that are ideal properties of biomarkers, including conservation, abundance, and stability in plasma, saliva, and urine [14]. Recently, there have been reports that circRNAs are abnormally expressed in tumor tissues and cells, and participate in the process of malignant progression [15-20]. CircRNAs have the capacity to serve as a microRNAs (miRNAs) sponge and block the inhibition of miRNAs on their target genes, which may be implicated in cancer malignant behavior [21-23].

Exosomes are nanoscale vesicles secreted by cells, which have attracted more and more attention in the past decades. Previous reports have shown that exosomes contain various biomolecules, such as proteins [24], lipids [25], and nucleic acids [26]. Exosomes can help to treat different types of cancer, inhibit the local and distant spread of the tumor, and have the potential of early detection of cancer and inhibition of drug resistance [27]. In addition, exosomes are always abnormally expressed in malignant progression and can be found in the early stage of cancer. It is well documented 
that exosomal RNAs could be promising clinical biomarkers for various cancers [28, 29]. Furthermore, tumor-derived exosomes play a role in signal transduction of intercellular communication [30-32]. In the process of malignant progression, tumor cells release exosomes and regulate the tumor microenvironment. In addition, tumor cells secrete exosomes, which change the composition of lung, liver, and other distal organs, and contribute to the distant metastasis of the tumor. Because the secreted or excreted exosomes, such as saliva, ascites, and cervicovaginal lavage fluid, can be evaluated noninvasively or minimally invasive. If exosomal RNAs can be used as a diagnostic, prognostic, or predictive biomarker, doctors can obtain samples from patients for diagnosis and prognosis through relatively noninvasive methods in the future. In the recent five years, exosomal RNAs as a clinical biomarker have achieved some promising results in various types of tumors.

However, the diagnostic value of plasma circRNAs for colorectal adenomas or early tumors is still unclear. In this study, we investigate the circRNAs' expression profiles in plasma exosomes from colorectal adenoma and healthy controls. We further explored the possible molecular mechanism associated with the differentially expressed circRNAs by GO analysis. Our work provides novel insight into novel potential biomarkers in the detection of colorectal adenomas or early tumors.

\section{Patients and methods}

Patients and plasma specimens. All samples used in this study were from our hospital (First Affiliated Hospital, Zhejiang University School of Medicine, Zhejiang, China). Diagnosis of colorectal adenoma was confirmed by histopathological analysis. None of the patients received preoperative chemotherapy or radiotherapy. EDTA blood tubes were used to collected plasma samples. Sample collection, use, and storage procedures used in this study were approved by the Ethics Committee of Zhejiang University.

Plasma exosome isolation. Exosomes were separated by the ExoQuick exosome precipitation kit (System Bioscience, Palo Alto, CA, USA), according to the manufacturer's directions. To be brief, the pelleted exosomes were resuspended in $1 \times$ phosphate-buffered saline (PBS) and their concentration was estimated by the Bradford assay. Exosomes were stored at $-80{ }^{\circ} \mathrm{C}$ prior to further use.

Western blotting. The expression of the exosomal markers (CD81 and CD63) was analyzed by western blotting. Protein concentration was measured using a BCA Protein Assay Kit (Pierce, Rockford, IL, USA). Proteins were denatured by heating at $90^{\circ} \mathrm{C}$ for $10 \mathrm{~min}$ in $4 \times$ nuPAGE LDs sample buffer (Life Technologies, Carlsbad, CA, USA). Equal amounts of protein from each sample were separated by $6-18 \%$ sodium dodecyl sulfate-polyacrylamide gel electrophoresis (SDS-PAGE) (Life Technologies). Then, the proteins were transferred to a polyvinylidene fluoride membrane (Milli- pore, Billerica, MA, USA). After blocking with $5 \%$ non-fat milk, the membrane was incubated overnight at $4{ }^{\circ} \mathrm{C}$ with the primary anti-CD81 antibody (1:1000, ab109201, Abcam, Cambridge, MA, USA) and anti-CD63 (1:1000, ab216130, Abcam, Cambridge, MA, USA). After washing three times with Tris-buffered saline with $0.05 \%$ Tween-20 for $10 \mathrm{~min}$ each, the signal was detected with the SuperSignal West Pico Chemiluminescent Substrate (Pierce).

Transmission electron microscopy. Exosomes were re-suspended in $200 \mu \mathrm{l}$ PBS and then this solution was transferred to a carbon-coated $\mathrm{Cu}$ grid (ProSciTech, Kirwan, QLD, Australia) and incubated at room temperature for 5 minutes. Exosomes were negatively stained with aqueous uranyl acetate of $2 \%$ for 2 minutes and washed three times in PBS. After drying, the morphologies of isolated exosomes were observed by transmission electron microscopy (TEM; JEOL JEM-1010, Japan) at room temperature.

RNA isolation, library construction, and RNA-seq analysis. High throughput sequencing was carried out by Cloud-Seq Biotech (Shanghai, China). We used the RiboZero rRNA Removal Kit (Illumina, San Diego, CA, USA) to remove the rRNA from total RNA following the manufacturer's instructions. The CircRNA Enrichment Kit (Cloudseq, USA) was used to enrich the circRNAs. RNA library was constructed with TruSeq Stranded Total RNA Library Prep Kit (Illumina, San Diego, CA, USA), and the quality and quantity of the library were controlled by the BioAnalyzer 2100 system (Agilent Technologies, Inc., Santa Clara, CA, USA). After libraries were transformed into single-stranded DNA molecules, the product was captured by Illumina and sequenced for 150 cycles on Illumina HiSeqTM 4000 Sequencer (Illumina, San Diego, CA, USA).

Reverse transcription-quantitative polymerase chain reaction (RT-qPCR). The expression of the candidate circRNAs was validated by qRT-PCR. Total RNA concentration was quantified by NanoDrop spectrophotometer (Thermo Fisher Scientific) at $260 \mathrm{~nm}$. Template complementary DNA (cDNA) was synthesized by PrimeScript RT Reagent Kit (Takara, Dalian, China) according to the manufacturer's instructions. The resultant cDNA was then used as a template for the real-time PCR amplification of the SYBR Premix Ex Taq II (Takara, Japan) operated on the Bio-Rad QX100 Droplet Digital PCR system (USA). Relative RNA amount was calculated by the $2^{\Delta \Delta \mathrm{Ct}}$ method with the normalization to GAPDH. The primers were as follows: GAPDH-Forward: GGGAGCCAAAAGGGTCATCA, and Reverse: TGATGGCATGGACTGTGGTC; hsa_circ_0004075-Forward: GGAACAGACTCCACCCAAACAT, and Reverse: TCATTGTCATCGTCATCACTGTC.

Statistical analysis. All data were presented as the mean \pm SD. Statistical analysis was processed by the Statistical Program for Social Sciences (SPSS) 22.0 software (SPSS, Chicago, IL, USA) and GraphPad Prism 5.0 (GraphPad Software, La Jolla, CA, USA). The unpaired Student t-test was used to analyze qRT-PCR results between the case group 
and the control group. The value of $\mathrm{p}<0.05$ was regarded as a statistically significant difference.

\section{Results}

Characterization of plasma exosomes. To determine the characteristics of the exosomes, we performed TEM, western blot, and NTA. Plasma exosomes from colorectal adenomas patients and healthy controls expressed abundant exosomal markers CD63 and CD81, as shown by western blot (Figure 1A). TEM showed that exosomes exhibited roundshaped morphologies with mean diameters around $76.89 \mathrm{~nm}$ (Figure 1B). The exosomes size distribution was measured by NTA. The NTA results illustrated that the concentration was $2.4 \mathrm{E}+7$ particles/nm and the mean size was $76.89 \mathrm{~nm}$ (Figure 1C). The above analysis indicated that the plasmaderived particles we isolated were exosomes.

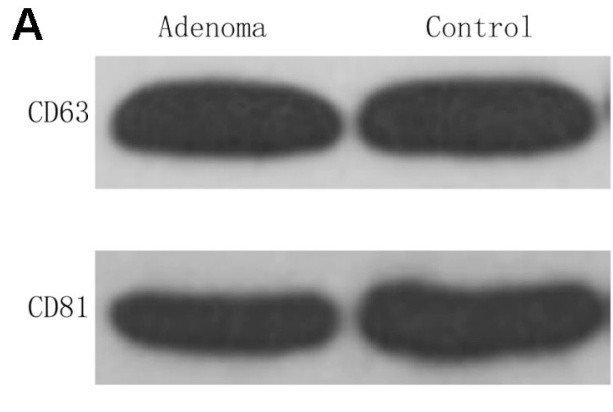

B

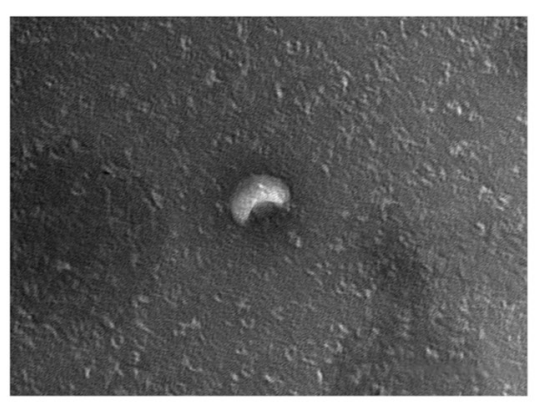

C

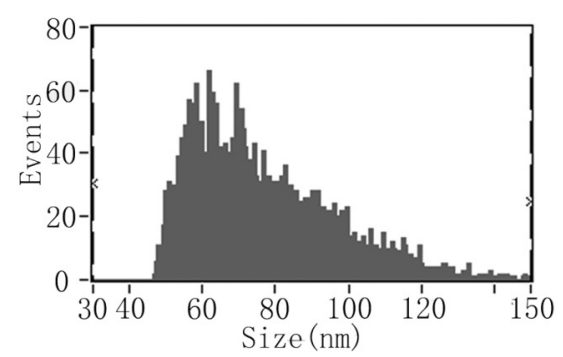

Figure 1. Identification of exosomes isolated from plasma. A) Exosomal markers $(\mathrm{CD} 63, \mathrm{CD} 81)$ in representative plasma exosomes were determined by western blot. B) The morphology and size of plasma exosomes detected by TEM. C) The size and concentration of serum exosomes measured by NTA. NTA, Nanoparticle Tracking Analysis; TEM, transmission electron microscopy.
Differential analysis of exosomal circRNAs. The circRNAs profiles in exosomes from plasma were analyzed by RNA deep sequencing. The circRNA expression profile of the colorectal adenomas and controls is shown in a hierarchical clustering heatmap (Figure 2A). A circRNA candidate was considered to be significantly different according to statistical criteria of fold change $\geq 2.0$ and $p$-value $\leq 0.05$. A scatter plot (Figure 2B) and a volcano plot (Figure 2C) of all the differentially expressed circRNAs exhibited the distinguishable circRNA expression profiles between colorectal adenomas patients and healthy controls. In total, among the 35,191 identified circRNAs, 112 were significantly upregulated and 301 were significantly downregulated in colorectal adenomas compared with controls (Figure 2D).

Exosomal circRNA profiling in the plasma. To further uncover the characteristics of the differentially expressed circRNAs, we classified them according to their different positions on chromosomes. Our results revealed that the circRNAs are situated at all of the human chromosomes (Figure 3A). Moreover, among these circRNAs, 11.62\% were intronic circRNAs, $66.59 \%$ were exonic circRNAs, $5.08 \%$ were antisense circRNAs, and $16.71 \%$ of the circRNAs were of other sources (Figure 3B). Among the 112 upregulated circRNAs, 35 were confirmed as novel circRNAs, 77 are listed in the circBase, but in the downregulated circRNAs, 184 were identified as novel, while 117 circRNAs had been collected in databases (Figure 3C). Moreover, the length of these circRNAs was investigated; the majority of the differentially expressed circRNAs were $<2,000$ nucleotides (nt) in length (Figure 3D). The top 10 significantly upregulated or downregulated circRNAs in exosomes were listed in Table 1.

Functional analysis of the differentially expressed circRNAs. GO and KEGG analysis were applied on their parental genes to predict their potential functions in our study. GO annotation analysis elucidates the enrichment terms of biological processes (BP), cellular components (CC), and molecular functions (MF), thus further revealing the biological functions of upregulated circRNAs. The most highly enriched GO terms in biological processes were "organelle organization" and "protein sumoylation" (Figure 4A). The most highly correlated cellular component was primarily involved with "intracellular" and "intracellular organelle" (Figure 4B). Based on molecular functions, "DNA binding" and "binding" are closely related to these circRNAs (Figure 4C). KEGG pathway analysis (Figure 4D) suggested that the upregulated circRNAs were involved in several biological pathways. Among these pathways, the GnRH signaling pathway and MARK signaling pathway are the most prominent, which may be related to the molecular mechanism of pathogenesis.

Validation of exosomal circRNAs expression and circRNA-miRNA-mRNA network. To further validate exosomal circRNA-seq results, the exosomes isolated from 12 pairs of samples (12 colorectal adenomas and 12 controls), were used to extract the RNA for qRT-PCR. The 
levels of hsa_circ_0004075 in colorectal adenomas patients were significantly higher than the controls (Figure 5A), which was consistent with the sequencing results and confirmed the elevated expression of hsa_circ_0004075 in the plasma exosomes of the patients. It was reported that circRNAs could serve as miRNAs sponges to regulate gene expression [33]. To further unveil the putative miRNAs in colorectal adenomas, target miRNAs and mRNAs of the
A

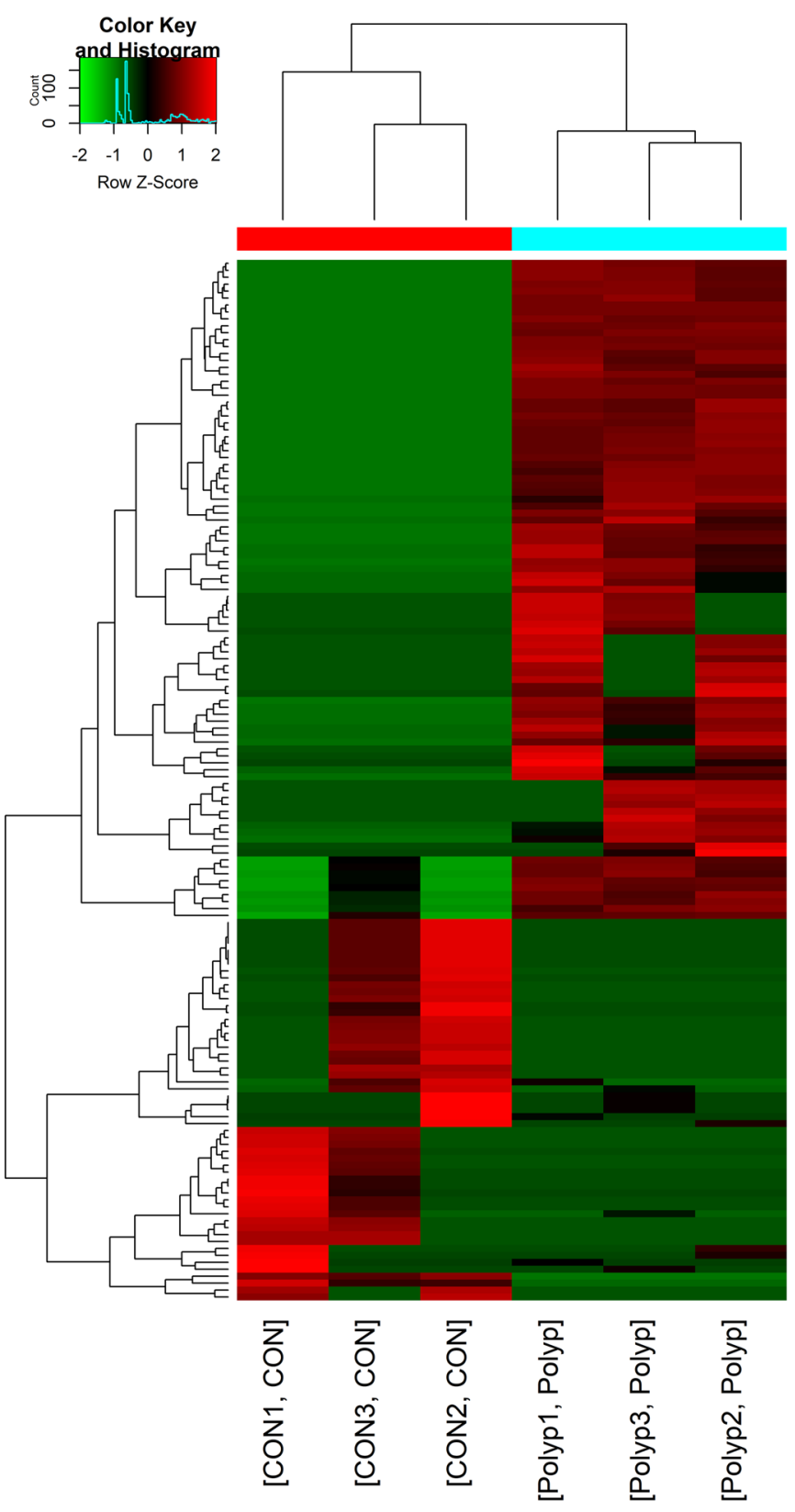

B

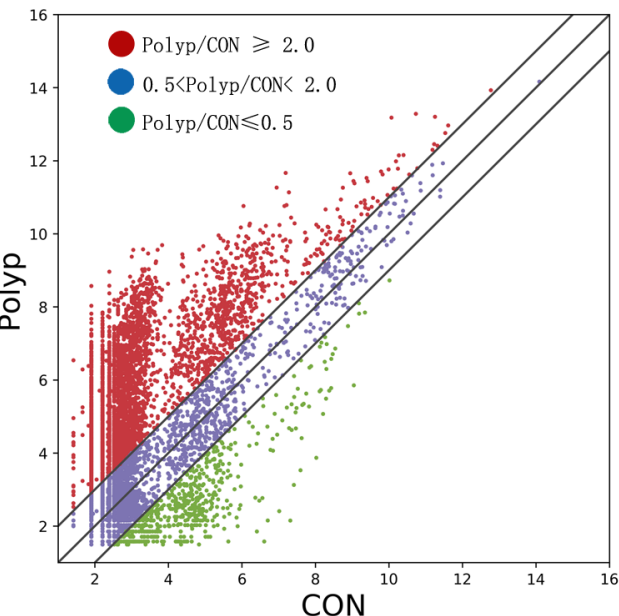

C

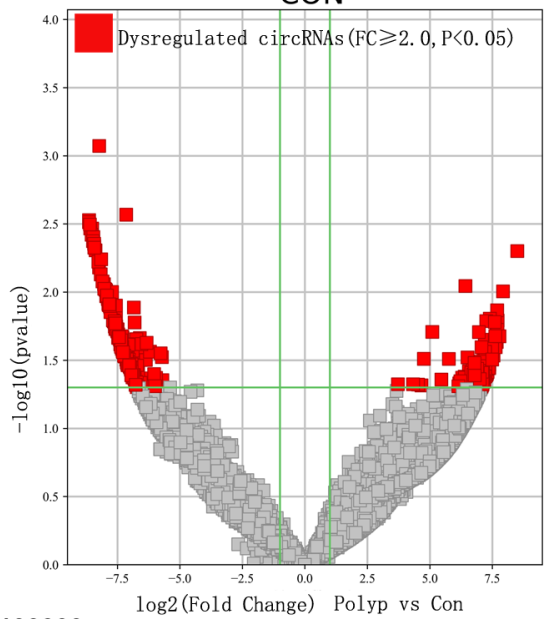

D

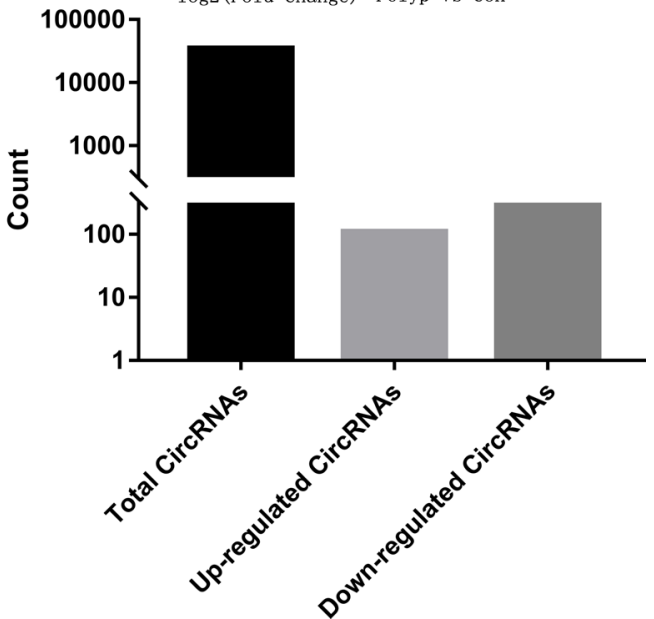

Figure 2. Differential analysis of exosomal circRNAs between colorectal adenomas patients and control individuals. A) Hierarchical clustering showed differences in circRNAs expression profiles between the two groups. B) The changes of circRNAs expression were visualized by scatter plot. C) The volcano plot revealed expression profiling between the two groups. D) The number of upregulated and downregulated circRNAs was displayed. 
Table 1. The top 10 exosomal circRNAs with downregulated and upregulated expression.

\begin{tabular}{|c|c|c|c|c|c|}
\hline CircRNA ID & $\log$ FC & p-value & CircBase ID & Gene name & Catalog \\
\hline chr2:121112155-121112311+ & -8.636884095 & 0.00307173 & & & intergenic \\
\hline chr6:136977445-137041727- & -8.636884095 & 0.00307173 & hsa_circ_0077977 & MAP3K5 & exonic \\
\hline chr7:7622749-7629194+ & -8.634455762 & 0.002968638 & & MIOS & exonic \\
\hline chr6:84922675-84925640- & -8.59841348 & 0.003196802 & hsa_circ_0077228 & CEP162 & exonic \\
\hline chr19:14023136-14024451+ & -8.585905697 & 0.0034144 & & CC2D1A & exonic \\
\hline chr1:57766077-57766273+ & -8.533072267 & 0.003798969 & & $\mathrm{DAB} 1$ & antisense \\
\hline chr1:235602084-235606226+ & -8.508983858 & 0.003418473 & hsa_circ_0112516 & TBCE & exonic \\
\hline chrM:13979-15632- & -8.478243893 & 0.004231869 & & JA760602 & sense overlapping \\
\hline chr1:28818162-28819603+ & -8.462067206 & 0.003950038 & hsa_circ_0011143 & PHACTR4 & exonic \\
\hline chr17:2726836-2740135+ & -8.432797171 & 0.00442625 & & RAP1GAP2 & intronic \\
\hline chr11:47774468-47776216- & 8.50022658 & 0.005000554 & hsa_circ_0004075 & FNBP4 & exonic \\
\hline chr12:110922883-110925748+ & 7.930254881 & 0.009884176 & hsa_circ_0004516 & FAM216A & exonic \\
\hline chr12:123497160-123498589- & 7.786277233 & 0.021099941 & hsa_circ_0029170 & PITPNM2 & exonic \\
\hline chr10:20436713-20466338+ & 7.717343152 & 0.016165229 & hsa_circ_0000224 & PLXDC2 & exonic \\
\hline chr17:60629663-60642498+ & 7.707548797 & 0.018901116 & hsa_circ_0008371 & TLK2 & exonic \\
\hline chr22:38917613-38964294- & 7.69510056 & 0.013608844 & hsa_circ_0001231 & $\mathrm{DMC1}$ & exonic \\
\hline chr4:83852096-83867627- & 7.679225451 & 0.023261962 & hsa_circ_0127102 & LIN54 & exonic \\
\hline chr18:67540380-67614669- & 7.666702322 & 0.018706079 & & CD226 & exonic \\
\hline chr5:94275788-94353188- & 7.632999255 & 0.025364699 & hsa_circ_0130059 & MCTP1 & exonic \\
\hline chr10:11978544-11994248- & 7.620493417 & 0.021040435 & hsa_circ_0017705 & UPF2 & exonic \\
\hline
\end{tabular}

A

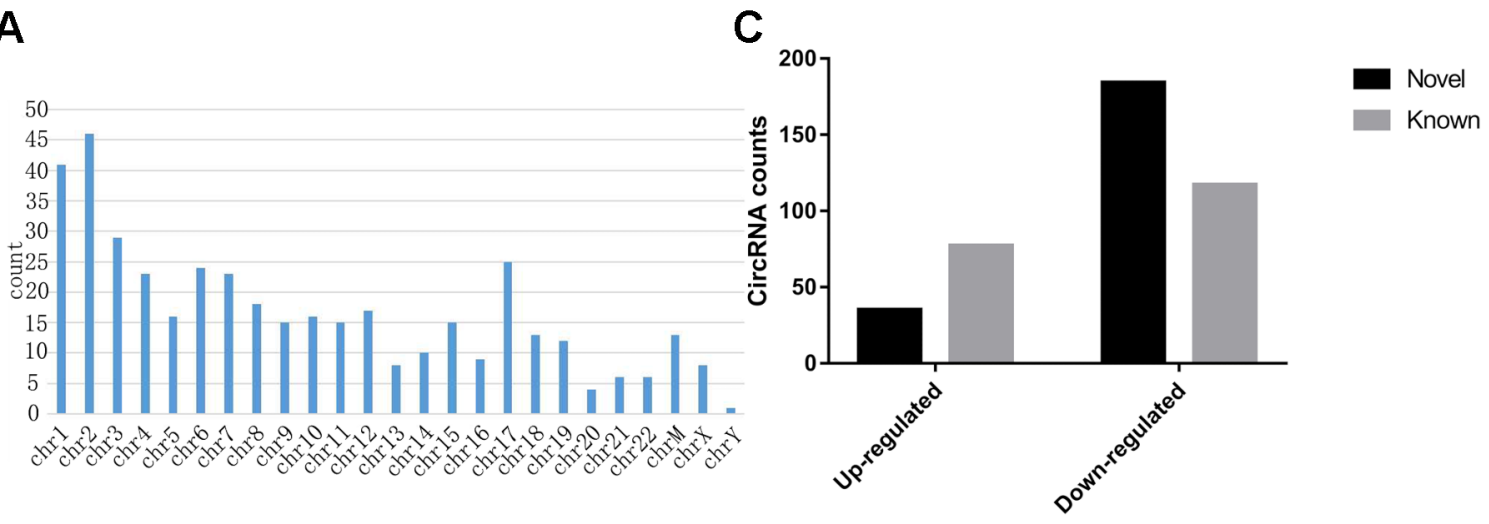

B

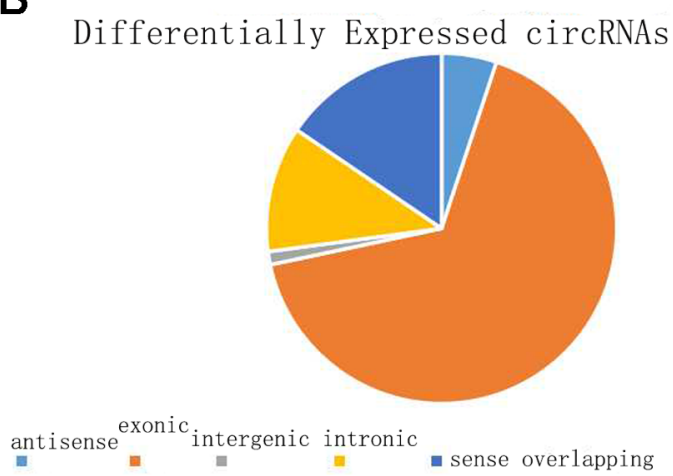

D

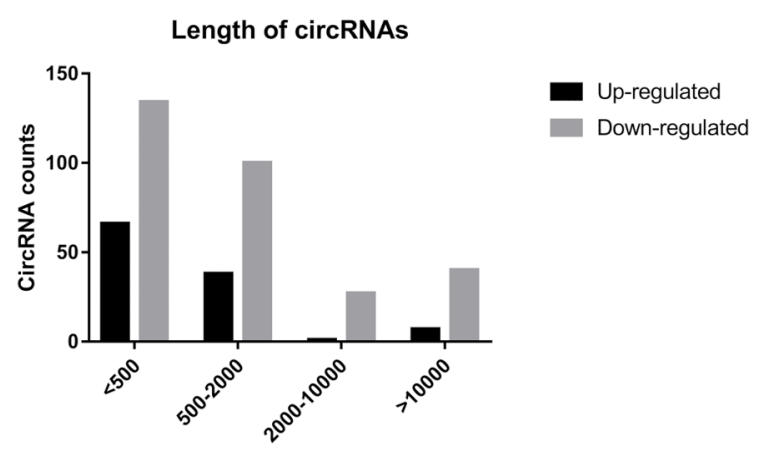

Figure 3. Profiling of circular RNAs in human serum exosomes. A) The distributions of the differentially expressed circRNAs in human chromosomes. B) Classification of circRNAs based on the genomic origin. C) Among the differentially expressed circRNAs, the novel and known circRNAs were uncovered. D) Length distribution of the circRNAs was unveiled. 
hsa_circ_0004075 were predicted by miRanda and Target Scan. And the top 5 putative binding miRNAs are hsa-miR6880-5p, hsa-miR-6511b-5p, hsa-miR-6851-5p, hsa-miR6740-5p, and hsa-miR-5004-3p. A circRNA-miRNA-target gene network for hsa_circ_0004075 was built by Cytoscape (Figure 5B).

\section{Discussion}

Colorectal cancer is still a very common cancer worldwide because it is the third most frequently diagnosed cancer in men and women [34, 35]. However, the underlying causes and molecular mechanisms of CRC remain to be understood. The occurrence and progression of CRC is a comprehensive process from benign adenoma to cancer. Alterations of key regulatory genes in the adenoma-carcinoma sequence mark the transition from normal to neoplasm. It was also found that adenoma removal significantly reduced the risk of death from colorectal cancer, as compared with that in the general population [36].

Early detection and complete resection of precursor lesions could effectively halt the polyp carcinogenesis [37, 38]. At present, colonoscopy is the widely used method of colorectal examination. Although it is considered the gold standard for diagnosis and treatment of adenoma, colonoscopy has its limitations and is user-dependent, such as diet restriction, bowel preparation, and well-trained examination. The polyp miss rate as determined by colonoscopy
A

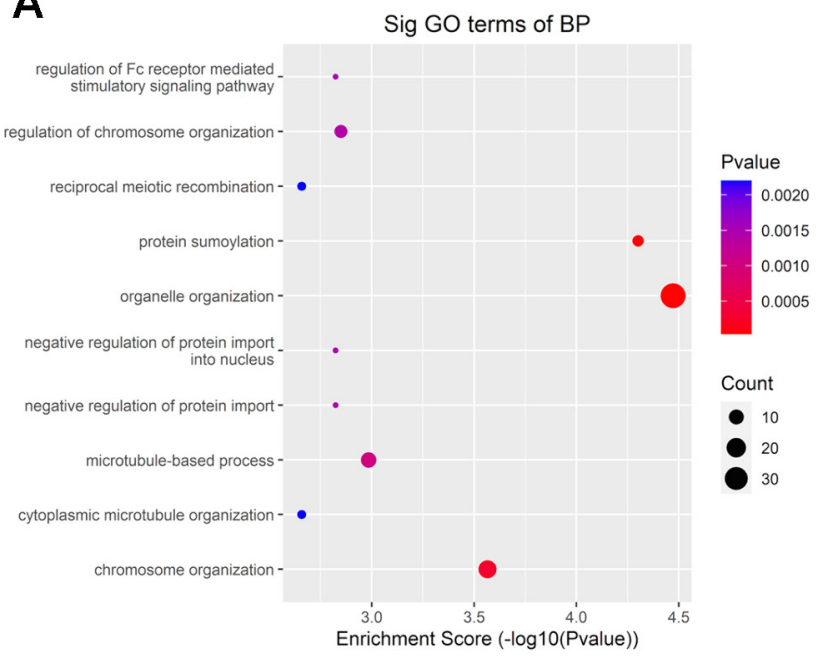

C

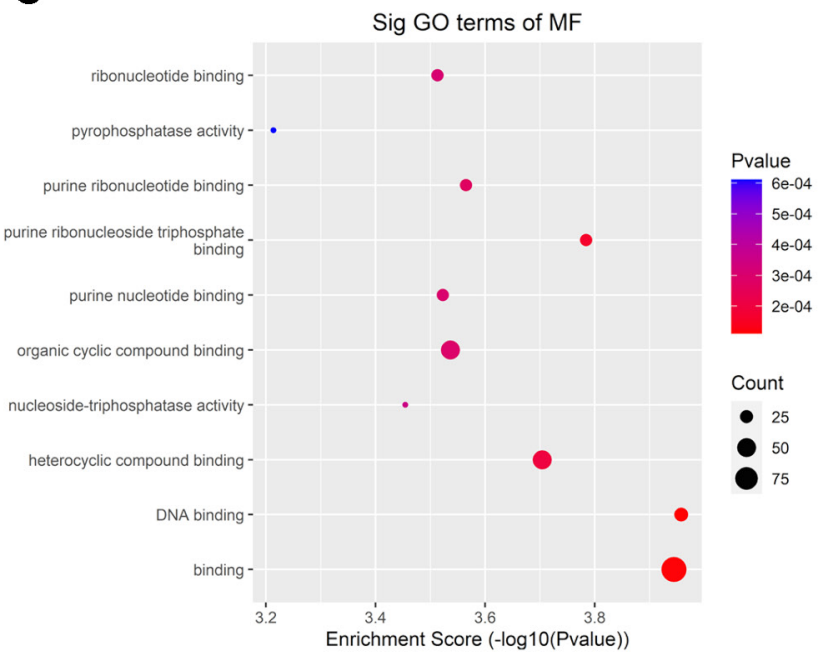

B

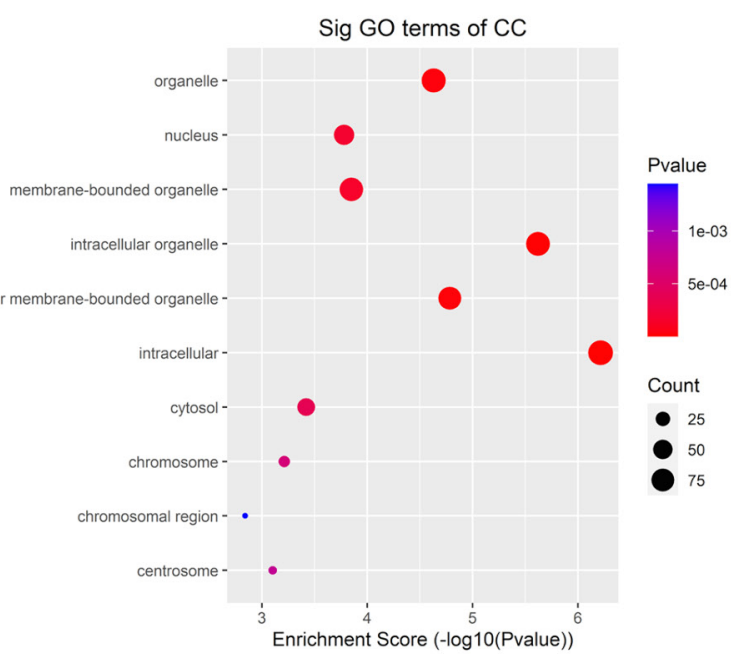

D

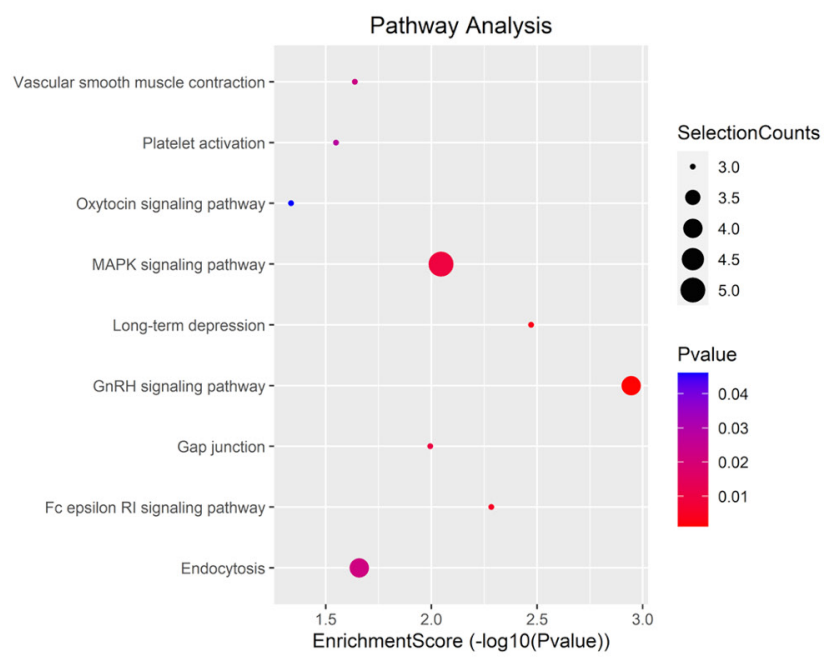

Figure 4. GO and KEGG analysis of significantly differential expression. The top 10 enriched A) biological processes, B) cellular components, and C) molecular functions were discovered by GO analysis. D) The most significantly enriched pathway was unveiled by KEGG analysis. 
is estimated between 20 and 22\% [39]. The noninvasive detection of adenoma is still under study. A multitarget stool DNA test was recommended as a new CRC screening method in the updated guideline for adults elder than 45 years with an average risk of CRC [40]. As a potential minimally invasive way, a liquid biopsy will play a greater role in clinical application. High-throughput sequencing has been applied to liquid biopsy. Exosomes could be released by cancer cells and act as intercellular mediators of oncogenic information [41]. Compelling evidence has indicated that cancer-derived exosomal miRNAs play an oncogenic role in CRC growth, chemoresistance, and metastasis, and could be potential diagnostic biomarkers for CRC [42-45]. Moreover, the levels of exosomal miRNAs were higher in several tumor cell lines compared to non-malignant cell lines in accordance with their findings in serum exosomes of CRC patients and healthy individuals $[46,47]$. Many studies have focused on ncRNAs in exosomes, and observed an upregulation of some ncRNAs in exosomes of cancer subjects when compared to healthy control $[48,49]$.

A

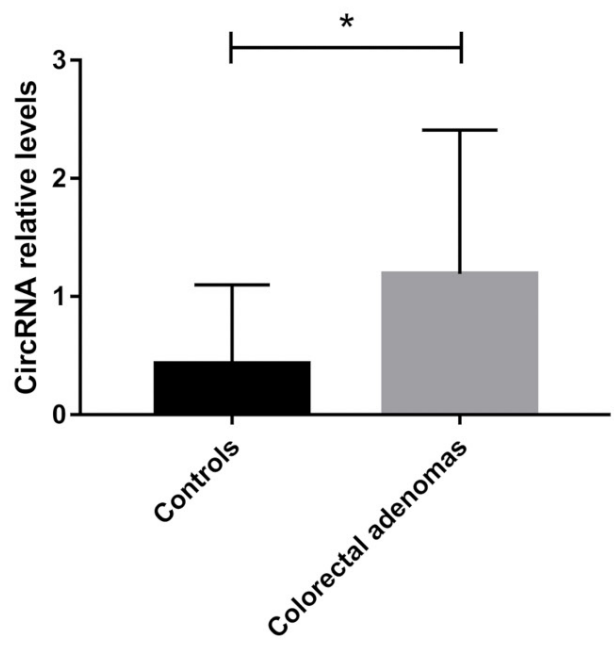

B

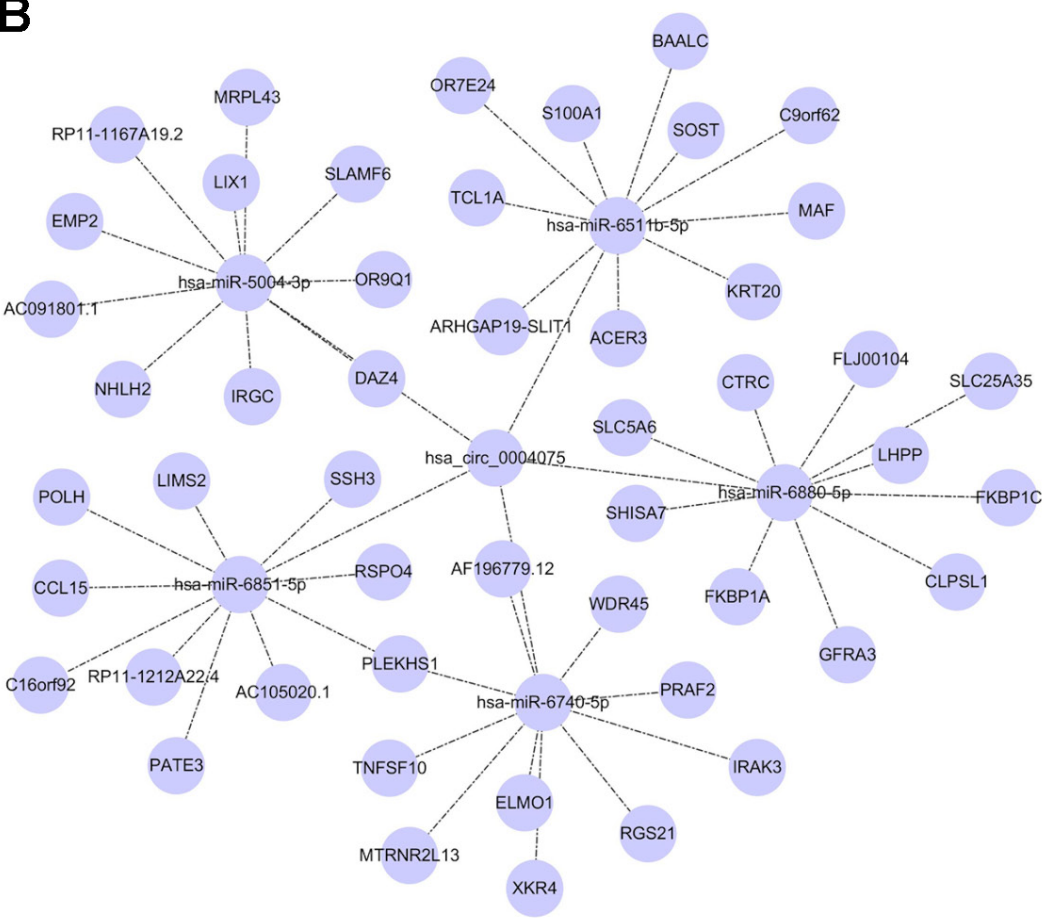

Figure 5. Prediction and analyses of circRNA-miRNA-mRNA network. A) Validation of the RNA sequencing results by qRT-PCR. B) The hsa circ_0004075-mediated ceRNA network. 
However, the role of exosomes in colorectal adenomas still needs to be further clarified.

Exosomes can be transferred from tumor cells to the peripheral circulation and act as carriers of information in intercellular communication $[50,51]$. The differentially expressed exosomal circRNAs in the plasma may play a pivotal role in affecting tumor growth and proliferation and mediating the crosstalk between the tumor and tumor-associated cells. To explore the mechanism of plasma exosomes in colorectal adenomas and healthy control, the exosomes were isolated from the plasma of 3 colorectal adenomas patients and 3 healthy individuals. We then identified these exosomes using TEM, WB, and NTA (Figures 1A-1C).

Alterations in components of exosomes in plasma are related to the pathological processes of various diseases, including cancer [52]. CircRNAs form a class of posttranscriptional regulators, which compete with other RNAs for binding by miRNAs and RBPs and may generally function in modulating the concentration of RBPs, RNAs, or their binding sites [53]. It was reported that the expression patterns of circRNAs varied between tumor and normal cells $[54,55]$. It is necessary to identify anti-degradation biomarkers for clinical screening analysis. Vo et al. have reported that circRNAs were more stable than linear RNA in plasma after incubation, and circRNAs could be reliably detected in urine samples [56]. These unique characteristics could present them as a promising biomarker for early detection and prognosis. Accumulating evidence showed that some circRNAs are involved in the malignant progression of colorectal cancer, including malignant proliferation, chemotherapy resistance, and metastasis [57]. Furthermore, the potential functions of circRNAs have been investigated extensively, including sponging miRNAs, encoding peptides, binding to RBPs, promoting gene transcription, and regulating alternative splicing [58, 59]. Additionally, miRNAs, lncRNAs, and circRNAs are harbored by released exosomes and play a crucial role in carcinogenesis as a messenger by carrying and delivering oncogenic molecules in cellular communication [60,61]. In recent years, more and more attention has been paid to the circRNAs of exosomes as diagnostic and therapeutic targets, and the expression profiles of some disease-related exosomes have been identified. Uratani et al. demonstrated the potential role of total serum miR-21, miR-29a, and miR-92a in the early detection of colorectal adenomas and cancers [62]. CircRNAs and exosomal circRNAs have the potential diagnostic values for gastrointestinal (GI) malignancies and lung cancer $[63,64]$. Nevertheless, the role of exosomal circRNAs in colorectal adenomas has not been established. In the present study, we have analyzed the clinical correlation of plasma exosomal circRNAs in colorectal adenomas compared to the healthy control group based on the results of the RNA sequence. Our results uncovered that 413 circRNAs were differentially expressed between colorectal adenomas patients and healthy control (Figure 2D).
New studies revealed that exosomal circRNAs are involved in the malignant transformation, and achieve the efficient transmission of phenotypical changes, thereby promoting malignant progression [65-67].

We carried out this research to identify an intracellular communication mechanism that hasn't been explained before in colorectal adenomas.

We focused on the characteristics of exosomal circRNAs that are significantly upregulated in colorectal adenomas, as they are likely to be associated with the progression. To investigate the role of the differentially expressed circRNAs in colorectal adenomas, we performed GO and KEGG pathway analyses to predict their potential functions. GO analysis uncovered that these circRNAs were significantly associated with the organelle organization, intracellular organelle, and DNA binding (Figures 3A-3D). Therefore, we suspected that these circRNAs might be related to the regulation of the signaling pathway. Similarly, the highly relevant signaling pathways are mainly involved in the GnRH signaling pathway and MARK signaling pathway from the KEGG pathway analyses.

The previous study has indicated that there are six means of circRNAs functions, including serving as miRNA sponges, protein sponges, protein function enhancers, protein scaffolds, protein recruiters, and translation templates [68]. The competitive endogenous RNA (ceRNA) hypothesis supposed that RNAs may affect miRNAs activity by competing miRNAs binding sites, thereby regulating miRNAs target gene expression [69]. There are various miRNA binding sites in circRNAs, which would attenuate miRNAs activity and upregulate the level of miRNAs target genes. It provides new insights into exploring the mechanisms of the circRNAs. In our study, the potential miRNA targets of hsa_circ_0004075 were predicted by the database. The results showed negative correlations between hsa circ_0004075 and related miRNAs. These results revealed that hsa_circ_0004075 may play a central role in the occurrence and development of colorectal cancer. Thus, further investigations are required to verify the utility of exosomal circRNAsHowever, it must be acknowledged that the sample size is not yet enough, which is the limitation of this study. In order to confirm the diagnostic efficiency of plasma exosomal hsa_circ_0004075, a large-scale, multi-center sample study is necessary. Due to our short study time and limitations on patient information, we did not find suitable data to explore the correlations between plasma exosomal hsa_circ_0004075 and adenoma recurrence. Therefore, it is necessary to establish a long-term follow-up system to demonstrate whether the level of plasma exosomal hsa circ_0004075 affects adenoma recurrence. We are confident that the current results indicated that plasma exosomal hsa circ_0004075 may be a potential biomarker for colorectal adenomas. In addition, qRT-PCR was used to detect the expression of exosomal hsa_circ_0004075 in plasma of fresh samples, which is convenient for clinical application. 
Collectively, based on our results, an initial landscape of the exosomal circRNAs differential expression in plasma from colorectal adenomas was presented through highthroughput sequencing and was validated using qRT-PCR. The results indicated that hsa_circ_0004075 has the potential as a novel biomarker and therapeutic target for colorectal adenomas. Our study also broadens the understanding of exosomal circRNAs in the pathogenesis of colorectal adenomas. Further research is needed to explore the underlying mechanisms of hsa_circ_0004075 in colorectal cancer evaluation in the future.

Acknowledgments: Our work is supported by the National Natural Science Foundation of China (81900461), and the Zhejiang Provincial Natural Science Foundation of China (LQ19H160022).

\section{References}

[1] BRAY F, FERLAY J, SOERJOMATARAM I, SIEGEL RL, TORRE LA et al. Global cancer statistics 2018: GLOBOCAN estimates of incidence and mortality worldwide for 36 cancers in 185 countries. CA Cancer J Clin 2018; 68: 394-424. https://doi.org/10.3322/caac. 21492

[2] HOLLESTEIN LM, VAN HERK-SUKEL MP, RUITER R, DE VRIES E, MATHIJSSEN RH et al. Incident cancer risk after the start of aspirin use: results from a Dutch populationbased cohort study of low dose aspirin users. Int J Cancer 2014; 135: 157-165. https://doi.org/10.1002/ijc.28634

[3] LADABAUM U, DOMINITZ JA, KAHI C, SCHOEN RE. Strategies for Colorectal Cancer Screening. Gastroenterology 2020; 158: 418-432. https://doi.org/10.1053/j.gastro.2019.06.043

[4] LHEWA DY, STRATE LL. Pros and cons of colonoscopy in management of acute lower gastrointestinal bleeding. World J Gastroenterol 2012; 18: 1185-1190. https://doi. org/10.3748/wjg.v18.i11.1185

[5] BINEFA G, RODRIGUEZ-MORANTA F, TEULE A, MEDINA-HAYAS M. Colorectal cancer: from prevention to personalized medicine. World J Gastroenterol 2014; 20: 67866808. https://doi.org/10.3748/wjg.v20.i22.6786

[6] SCHREUDERS EH, GROBBEE EJ, SPAANDER MC, KUIPERS EJ. Advances in Fecal Tests for Colorectal Cancer Screening. Curr Treat Options Gastroenterol 2016; 14: 152162. https://doi.org/10.1007/s11938-016-0076-0

[7] QU SB, YANG XS, LI XL, WANG JL, GAO Y et al. Circular RNA: A new star of noncoding RNAs. Cancer Lett 2015; 365: 141-148. https://doi.org/10.1016/j.canlet.2015.06.003

[8] WANG YM, MO YZ, GONG ZJ, YANG X, YANG M et al. Circular RNAs in human cancer. Mol Cancer 2017; 16: 25. https://doi.org/10.1186/s12943-017-0598-7

[9] LASDA E, PARKER R. Circular RNAs: diversity of form and function. RNA 2014; 20: 1829-1842. https://doi.org/10.1261/ rna.047126.114

[10] VICENS Q, WESTHOF E. Biogenesis of Circular RNAs. Cell 2014; 159: 13-14. https://doi.org/10.1016/j.cell.2014.09.005
[11] LI J, SUN D, PU WC, WANG J, PENG Y. Circular RNAs in Cancer: Biogenesis, Function, and Clinical Significance. Trends Cancer 2020; 6: 319-336. https://doi.org/10.1016/j. trecan.2020.01.012

[12] JECK WR, SHARPLESS NE. Detecting and characterizing circular RNAs. Nat Biotechnol 2014; 32: 453-461. https:// doi.org/10.1038/nbt.2890

[13] MEMCZAK S, JENS M, ELEFSINIOTI A, TORTI F, KRUEGER J et al. Circular RNAs are a large class of animal RNAs with regulatory potency. Nature 2013; 495: 333-338. https://doi.org/10.1038/nature11928

[14] BROWN JR, CHINNAIYAN AM. The Potential of Circular RNAs as Cancer Biomarkers. Cancer Epidemiol Biomarkers Prev 2020; 29: 2541-2555. https://doi.org/10.1158/10559965.EPI-20-0796

[15] WANG LY, LONG HY, ZHENG QH, BO XT, XIAO XH et al. Circular RNA circRHOT1 promotes hepatocellular carcinoma progression by initiation of NR2F6 expression. Mol Cancer 2019; 18: 119. https://doi.org/10.1186/s12943-0191046-7

[16] CHENG ZA, YU CT, CUI SH, WANG H, JIN HJ et al. circTP63 functions as a ceRNA to promote lung squamous cell carcinoma progression by upregulating FOXM1. Nat Commun 2019; 10: 3200. https://doi.org/10.1038/s41467019-11162-4

[17] WANG S, ZHANG X, LI Z, WANG WZ, LI BW et al. Circular RNA profile identifies circOSBPL10 as an oncogenic factor and prognostic marker in gastric cancer. Oncogene 2019; 38: 6985-7001. https://doi.org/10.1038/s41388-019-0933-0

[18] XU JZ, SHAO CC, WANG XJ, ZHAO X, CHEN JQ et al. circTADA2As suppress breast cancer progression and metastasis via targeting miR-203a-3p/SOCS3 axis. Cell Death Dis 2019; 10: 175. https://doi.org/10.1038/s41419-019-1382-y

[19] LU Q, LIU TY, FENG HJ, YANG R, ZHAO XZ et al. Circular RNA circSLC8A1 acts as a sponge of miR-130b/miR494 in suppressing bladder cancer progression via regulating PTEN. Mol Cancer 2019; 18: 111. https://doi.org/10.1186/ s12943-019-1040-0

[20] WANG RJ, ZHANG S, CHEN XY, LI N, LI JW et al. CircNT5E Acts as a Sponge of miR-422a to Promote Glioblastoma Tumorigenesis. Cancer Res 2018; 78: 4812-4825. https://doi.org/10.1158/0008-5472.CAN-18-0532

[21] DING W, SHI Y, ZHANG H. Circular RNA circNEURL4 inhibits cell proliferation and invasion of papillary thyroid carcinoma by sponging miR-1278 and regulating LATS1 expression. Am J Transl Res 2021; 13: 5911-5927

[22] WEN C, FENG X, YUAN $\mathrm{H}$, GONG $\mathrm{Y}$, WANG G. Circ_0003266 sponges miR-503-5p to suppress colorectal cancer progression via regulating PDCD4 expression. BMC Cancer 2021; 21: 284. https://doi.org/10.1186/s12885-02107997-0

[23] WANG P, ZHOU C, LI D, ZHANG D, WEI L et al. circMTO1 sponges microRNA-219a-5p to enhance gallbladder cancer progression via the TGF-beta/Smad and EGFR pathways. Oncol Lett 2021; 22: 563. https://doi.org/10.3892/ ol.2021.12824 
[24] SIMPSON RJ, LIM JW, MORITZ RL, MATHIVANAN S. Exosomes: proteomic insights and diagnostic potential. Expert Rev Proteomics 2009; 6: 267-283. https://doi. org/10.1586/epr.09.17

[25] VIDAL M, SAINTE-MARIE J, PHILIPPOT JR, BIENVENUE A. Asymmetric distribution of phospholipids in the membrane of vesicles released during in vitro maturation of guinea pig reticulocytes: evidence precluding a role for "aminophospholipid translocase". J Cell Physiol 1989; 140: 455-462. https://doi.org/10.1002/jcp.1041400308

[26] VALADI H, EKSTROM K, BOSSIOS A, SJOSTRAND M, LEE JJ etal.Exosome-mediated transfer ofmRNAs and microRNAs is a novel mechanism of genetic exchange between cells. Nat Cell Biol 2007; 9: 654-659. https://doi.org/10.1038/ncb1596

[27] TANG ZY, LI DP, HOU SP, ZHU X. The cancer exosomes: Clinical implications, applications and challenges. Int J Cancer 2020; 146: 2946-2959. https://doi.org/10.1002/ijc.32762

[28] FAN LY, CAO QA, LIU J, ZHANG JP, LI BS. Circular RNA profiling and its potential for esophageal squamous cell cancer diagnosis and prognosis. Mol Cancer 2019; 18: 16. https://doi.org/10.1186/s12943-018-0936-4

[29] JIN XC, CHEN YF, CHEN HB, FEI SR, CHEN DD et al. Evaluation of Tumor-Derived Exosomal miRNA as Potential Diagnostic Biomarkers for Early-Stage Non-Small Cell Lung Cancer Using Next-Generation Sequencing. Clin Cancer Res 2017; 23: 5311-5319. https://doi.org/10.1158/1078-0432. CCR-17-0577

[30] DIOUFA N, CLARK AM, MA B, BECKWITT CH, WELLS A. Bi-directional exosome-driven intercommunication between the hepatic niche and cancer cells. Mol Cancer 2017; 16: 172. https://doi.org/10.1186/s12943-017-0740-6

[31] MILANE L, SINGH A, MATTHEOLABAKIS G, SURESH M, AMIJI MM. Exosome mediated communication within the tumor microenvironment. J Control Release 2015; 219: 278-294. https://doi.org/10.1016/j.jconrel.2015.06.029

[32] SUNG BH, WEAVER AM. Exosome secretion promotes chemotaxis of cancer cells. Cell Adh Migr 2017; 11: 187-195. https://doi.org/10.1080/19336918.2016.1273307

[33] CHEN YW, DU QR, HE YJ, CHEN WS, JIANG WY et al. Circ_0044516 Regulates miR-136/MAT2A Pathway to Facilitate Lung Cancer Development. J Immunol Res 2021; 2021: 5510869. https://doi.org/10.1155/2021/5510869

[34] SIEGEL RL, MILLER KD, JEMAL A. Cancer statistics, 2020. CA Cancer J Clin 2020; 70: 7-30. https://doi.org/10.3322/ caac. 21590

[35] SIEGEL RL, MILLER KD, FEDEWA SA, AHNEN DJ, MEESTER RG et al. Colorectal cancer statistics, 2017. CA Cancer J Clin 2017; 67: 177-193. https://doi.org/10.3322/ caac. 21395

[36] ZAUBER AG, WINAWER SJ, O’BRIEN MJ, VOGELAAR IL, BALLEGOOIJEN MV et al. Colonoscopic polypectomy and long-term prevention of colorectal-cancer deaths. N Engl J Med 2012; 366: 687-696. https://doi.org/10.1056/NEJMoa1100370

[37] JACOB BJ, MOINEDDIN R, SUTRADHAR R, BAXTER NN, URBACH DR. Effect of colonoscopy on colorectal cancer incidence and mortality: an instrumental variable analysis. Gastrointest Endoscop 2012; 76: 355-364. https://doi. org/10.1016/j.gie.2012.03.247
[38] LEVIN B, LIEBERMAN DA, MCFARLAND B, ANDREWS $\mathrm{KS}, \mathrm{BROOKS} \mathrm{D}$ et al. Screening and surveillance for the early detection of colorectal cancer and adenomatous polyps, 2008: a joint guideline from the American Cancer Society, the US Multi-Society Task Force on Colorectal Cancer, and the American College of Radiology. Gastroenterology 2008; 134: 1570-1595. https://doi.org/10.1053/j.gastro.2008.02.002

[39] VAN RIJN JC, REITSMA JB, STOKER J, BOSSUYT PM, VAN DEVENTER SJ et al. Polyp miss rate determined by tandem colonoscopy: a systematic review. Am J Gastroenterol 2006; 101: 343-350. https://doi.org/10.1111/j.15720241.2006.00390.x

[40] WOLF A, FONTHAM E, CHURCH TR, FLOWERS CR, GUERRA CE et al. Colorectal cancer screening for averagerisk adults: 2018 guideline update from the American Cancer Society. CA Cancer J Clin 2018; 68: 250-281. https://doi. org/10.3322/caac. 21457

[41] POKHAREL D, WIJESINGHE P, OENARTO V, LU J, SAMPSON DD et al. Deciphering Cell-to-Cell Communication in Acquisition of Cancer Traits: Extracellular Membrane Vesicles Are Regulators of Tissue Biomechanics. OMICS 2016; 20: 462-469. https://doi.org/10.1089/omi.2016.0072

[42] ZHAO SL, MI YS, GUAN BJ, ZHENG BB, WEI P et al. Tumor-derived exosomal miR-934 induces macrophage M2 polarization to promote liver metastasis of colorectal cancer. J Hematol Oncol 2020; 13: 156. https://doi.org/10.1186/ s13045-020-00991-2

[43] YAO SR, YIN Y, JIN GY, LI D, LI M et al. Exosome-mediated delivery of miR-204-5p inhibits tumor growth and chemoresistance. Cancer Med 2020; 9: 5989-5998. https://doi. org/10.1002/cam4.3248

[44] JIANG YM, JI XP, LIU K, SHI YQ, WANG CG et al. Exosomal miR-200c-3p negatively regulates the migration and invasion of lipopolysaccharide (LPS)-stimulated colorectal cancer (CRC). BMC Mol Cell Biol 2020; 21: 48. https://doi. org/10.1186/s12860-020-00291-0

[45] ZHANG Y, WANG SC, LAI QH, FANG YX, WU CJ et al. Cancer-associated fibroblasts-derived exosomal miR-17-5p promotes colorectal cancer aggressive phenotype by initiating a RUNX3/MYC/TGF-betal positive feedback loop. Cancer Lett 2020; 491: 22-35. https://doi.org/10.1016/j.canlet.2020.07.023

[46] ZENG ZC, LI YL, PAN YJ, LAN XL, SONG FY et al. Cancerderived exosomal miR-25-3p promotes pre-metastatic niche formation by inducing vascular permeability and angiogenesis. Nat Commun 2018; 9: 5395. https://doi.org/10.1038/ s41467-018-07810-w

[47] OGATA-KAWATA H, IZUMIYA M, KURIOKA D, HONMA Y, YAMADA Y et al. Circulating exosomal microRNAs as biomarkers of colon cancer. Plos One 2014; 9: e92921. https://doi.org/10.1371/journal.pone.0092921

[48] BARBAGALLO C, BREX D, CAPONNETTO A, CIRNIGLIARO M, SCALIA $M$ et al. LncRNA UCA1, upregulated in CRC Biopsies and Downregulated in Serum Exosomes, Controls mRNA Expression by RNA-RNA Interactions. Mol Ther Nucleic Acids 2018; 12: 229-241. https://doi.org/10.1016/j. omtn.2018.05.009 
[49] DONG L, LIN WR, QI P, XU MD, WU XB et al. Circulating Long RNAs in Serum Extracellular Vesicles: Their Characterization and Potential Application as Biomarkers for Diagnosis of Colorectal Cancer. Cancer Epidemiol Biomarkers Prev 2016; 25: 1158-1166. https://doi.org/10.1158/10559965.EPI-16-0006

[50] GONZALEZ-VILLASANA V, RASHED MH, GONZALEZCANTU Y, BAYRAKTAR R, MENCHACA-ARREDONDO JL et al. Presence of Circulating miR-145, miR-155, and miR-382 in Exosomes Isolated from Serum of Breast Cancer Patients and Healthy Donors. Dis Markers 2019; 2019: 6852917. https://doi.org/10.1155/2019/6852917

[51] MAIA J, CAJA S, STRANO MM, COUTO N, COSTA-SILVA B. Exosome-Based Cell-Cell Communication in the Tumor Microenvironment. Front Cell Dev Biol 2018; 6: 18. https:// doi.org/10.3389/fcell.2018.00018

[52] LI WH, LI CY, ZHOU T, LIU XH, LIU XN et al. Role of exosomal proteins in cancer diagnosis. Mol Cancer 2017; 16: 145. https://doi.org/10.1186/s12943-017-0706-8

[53] MEMCZAK S, JENS M, ELEFSINIOTI A, TORTI F, KRUEGER J et al. Circular RNAs are a large class of animal RNAs with regulatory potency. Nature 2013; 495: 333-338. https://doi.org/10.1038/nature11928

[54] WEI YP, CHEN X, LIANG C, LING Y, YANG XW et al. A Noncoding Regulatory RNAs Network Driven by CircCDYL Acts Specifically in the Early Stages Hepatocellular Carcinoma. Hepatology 2020; 71: 130-147. https://doi. org/10.1002/hep.30795

[55] CHEN SJ, HUANG V, XU X, LIVINGSTONE J, SOARES F et al. Widespread and Functional RNA Circularization in Localized Prostate Cancer. Cell 2019; 176: 831-843. https://doi. org/10.1016/j.cell.2019.01.025

[56] VO JN, CIESLIK M, ZHANG YJ, SHUKLA S, XIAO LB et al. The Landscape of Circular RNA in Cancer. Cell 2019; 176: 869-881. https://doi.org/10.1016/j.cell.2018.12.021

[57] TIAN J, XI X, WANG J, YU J, HUANG Q et al. CircRNA hsa_circ_0004585 as a potential biomarker for colorectal cancer. Cancer Manag Res 2019; 11: 5413-5423. https://doi. org/10.2147/CMAR.S199436

[58] REN CM, LIU J, ZHENG BX, YAN P, SUN YR et al. The circular RNA circ-ITCH acts as a tumour suppressor in osteosarcoma via regulating miR-22. Artif Cells Nanomed Biotechnol 2019; 47: 3359-3367. https://doi.org/10.1080/21691 401.2019.1649273

[59] ZENG KX, CHEN XX, XU M, LIU XX, HU XX et al. CircHIPK3 promotes colorectal cancer growth and metastasis by sponging miR-7. Cell Death Dis 2018; 9: 417. https://doi. org/10.1038/s41419-018-0454-8
[60] NEDAEINIA R, MANIAN M, JAZAYERI MH, RANJBAR $\mathrm{M}$, SALEHI $\mathrm{R}$ et al. Circulating exosomes and exosomal microRNAs as biomarkers in gastrointestinal cancer. Cancer Gene Ther 2017; 24: 48-56. https://doi.org/10.1038/ cgt.2016.77

[61] SUNDARARAJAN V, SARKAR FH, RAMASAMY TS. The multifaceted role of exosomes in cancer progression: diagnostic and therapeutic implications [corrected]. Cell Oncol (Dordr) 2018; 41: 223-252. https://doi.org/10.1007/s13402018-0378-4

[62] URATANI R, TOIYAMA Y, KITAJIMA T, KAWAMURA M, HIRO J et al. Diagnostic Potential of Cell-Free and Exosomal MicroRNAs in the Identification of Patients with High-Risk Colorectal Adenomas. Plos One 2016; 11: e160722. https:// doi.org/10.1371/journal.pone.0160722

[63] WANG Y, LI Z, XU S, GUO J. Novel potential tumor biomarkers: Circular RNAs and exosomal circular RNAs in gastrointestinal malignancies. J Clin Lab Anal 2020; 34: e23359. https://doi.org/10.1002/jcla.23359

[64] WANG Y, LU LJ, DUAN Y, LIU X, MAO Y et al. Analysis of circular RNA expression profiles of lung cancer in $\mathrm{Xu}-$ anwei, China. J Clin Lab Anal 2020; 34: e23521. https://doi. org/10.1002/jcla.23521

[65] DAI XY, CHEN C, YANG QL, XUE JC, CHEN X et al. Exosomal circRNA_100284 from arsenite-transformed cells, via microRNA-217 regulation of EZH2, is involved in the malignant transformation of human hepatic cells by accelerating the cell cycle and promoting cell proliferation. Cell Death Dis 2018; 9: 454. https://doi.org/10.1038/s41419-018-0485-1

[66] ZHANG HY, ZHU L, BAI M, LIU Y, ZHAN Y et al. Exosomal circRNA derived from gastric tumor promotes white adipose browning by targeting the miR-133/PRDM16 pathway. Int J Cancer 2019; 144: 2501-2515. https://doi.org/10.1002/ ijc.31977

[67] ZHANG HY, DENG T, GE SH, LIU Y, BAI M et al. Exosome circRNA secreted from adipocytes promotes the growth of hepatocellular carcinoma by targeting deubiquitinationrelated USP7. Oncogene 2019; 38: 2844-2859. https://doi. org/10.1038/s41388-018-0619-Z

[68] KRISTENSEN LS, ANDERSEN MS, STAGSTED L, EBBESEN KK, HANSEN TB et al. The biogenesis, biology and characterization of circular RNAs. Nat Rev Genet 2019; 20: 675-691. https://doi.org/10.1038/s41576-019-0158-7

[69] THOMSON DW, DINGER ME. Endogenous microRNA sponges: evidence and controversy. Nat Rev Genet 2016; 17: 272-283. https://doi.org/10.1038/nrg.2016.20 